\title{
The Efficacy and Safety of Acellular Matrix Therapy for Diabetic Foot Ulcers: A Meta-Analysis of Randomized Clinical Trials
}

\author{
Wentao Huang $\mathbb{D}^{1,2}$ Yongsong Chen, ${ }^{1}$ Nasui Wang, ${ }^{1}$ Guoshu Yin $\mathbb{D}^{1},{ }^{1}$ Chiju Wei, ${ }^{3}$ \\ and Wencan $\mathrm{Xu}$ iD ${ }^{1}$ \\ ${ }^{1}$ Department of Endocrinology and Metabolism, The First Affiliated Hospital of Shantou University Medical College, \\ 57 Changping Road, Shantou 515041, China \\ ${ }^{2}$ Shantou University Medical College, 22 Xinling Road, Shantou 515041, China \\ ${ }^{3}$ Multidisciplinary Research Center, Shantou University, 243 Daxue Road, Shantou 515063, China
}

Correspondence should be addressed to Wencan Xu; xuwcan@163.com

Received 21 November 2019; Accepted 3 January 2020; Published 1 February 2020

Academic Editor: Janet H. Southerland

Copyright (C) 2020 Wentao Huang et al. This is an open access article distributed under the Creative Commons Attribution License, which permits unrestricted use, distribution, and reproduction in any medium, provided the original work is properly cited.

Background. Acellular matrix (AM) therapy has shown promise in the treatment of diabetic foot ulcers (DFUs) in several studies. The clinical effects of AM therapy were not well established. Therefore, we conducted a meta-analysis of randomized clinical trials (RCTs) to examine the efficacy and safety of AM therapy for patients with DFUs. Methods. A literature search of 5 databases was performed to identify RCTs comparing AM therapy to standard therapy (ST) in patients with DFUs. The primary outcome was the complete healing rate and the secondary outcomes mainly included time to complete healing and adverse events. Results. Nine RCTs involving 897 patients were included. Compared with ST group, patients allocated to AM group had a higher complete healing rate both at 12 weeks (risk ratio $(R R)=1.73,95 \%$ confidence interval $(\mathrm{CI}): 1.31$ to 2.30 ) and 16 weeks $(\mathrm{RR}=1.56,95 \%$ CI: 1.28 to 1.91 ), a shorter time to complete healing (mean difference $(\mathrm{MD})=-2.41$; $95 \% \mathrm{CI}:-3.49$ to -1.32 ), and fewer adverse events $(\mathrm{RR}=0.64,95 \% \mathrm{CI}: 0.44$ to 0.93$)$. Conclusion. The present study suggests that AM therapy as an adjuvant treatment could further promote the healing of full-thickness, noninfected, and nonischemia DFUs. AM therapy also has a safety profile. More large well-designed randomized clinical trials with long follow-up duration are needed to further explore the efficacy and safety of AM therapy for DFUs.

\section{Introduction}

Diabetic mellitus, a rapid worldwide epidemic disorder, has become a major global health issue [1]. It is estimated that there are 451 million people with diabetes in 2017, and this number will rise to 693 million people by 2045 [2]. Diabetic foot ulcers (DFUs) are one of the most serious complications of diabetes and account for high levels of morbidity, mortality, and health-care costs [3-5]. The prevalence of DFUs is about $6.3 \%$ worldwide, and $19-34 \%$ of diabetic patients are liable to suffer from a foot ulceration in their lifetimes $[6,7]$. The standard therapy (ST) for DFUs includes debridement, dressing, offloading, vascular assessment, and infection and glycemic control [8]. However, the complete healing rates at 12 and 20 weeks are only $24 \%$ and $31 \%$, respectively, for those receiving ST [9]. In addition to ST in DFU care, there are a series of adjuvant therapies being studied, such as acellular matrix (AM) therapy, hyperbaric oxygen therapy, and shockwave therapy [8].

Acellular matrices (AMs) have been used as soft tissue replacement since 1994 [10]. The acellular grafts are processed to remove the cellular components while preserving the three-dimensional structure and the bioactive agents of extracellular matrix, such as collagen, hyaluronic acid, elastin, and fibronectin [11, 12]. Such matrices accelerate ulcer healing by providing structural supports and signals for cellular migration, proliferation, angiogenesis, and endogenous matrix production $[13,14]$. Several clinical trials have reported that AM therapy represents a useful adjuvant treatment for DFUs. However, reliable evidences on the 
clinical effects of AM therapy remain to be addressed. Therefore, the aim of this study was to evaluate the efficacy and safety of AM therapy for DFUs.

\section{Materials and Methods}

The meta-analysis was conducted according to the Preferred Reporting Items for Systematic Reviews and Meta-Analyses (PRISMA) statement [15].

2.1. Data Sources and Searches. A comprehensive literature search of PubMed, Embase, the Cochrane Library, and China Biology Medicine disc was performed by two independent researchers (W. H. and Y. C.) to identify RCTs assessing the efficacy and safety of AM in the treatment of DFUs. The last search update was conducted on September 24, 2019. A combination of Medical Subject Terms and keywords to define the concept of diabetic foot and acellular matrix was used, such as "diabetic foot, diabetic ulcer, diabetic wound, foot ulcer, diabetic" and "acellular dermis, acellular matrix, acellular tissue, acellular transplant, acellular graft, decellularized scaffold". There were no restrictions to language and publication date. In addition, the ClinicalTrial.gov database and reference lists in the selected articles were also searched for any eligible trials and information.

2.2. Study Selection. For inclusion in this meta-analysis, literatures needed to meet the following criteria: (1) RCTs consisted of more than 10 patients per group; (2) patients with type 1 or type 2 diabetes suffering from DFUs; (3) controlled trials examining AM therapy versus ST, such as debridement, dressing, offloading, antibiotic treatment, and glycemic control; and (4) studies reporting one of the outcomes at least, including complete healing rate, time to complete heal, ulcer area reduction, ulcer depth reduction, adverse events, and quality of life. The article with the most comprehensive data was included if there were duplicate studies from the same trial. Studies were excluded for the following reasons: (1) reviews, meta-analyses, conference abstracts without available full texts, letters, case reports, trials' protocol, retrospective studies, and animal studies; (2) standard therapy was not the control group or included other experimental treatments, such as growth factor treatment; (3) studies lacking control group; and (4) studies lacking sufficient data of interest. The selection of eligible studies from retrieved articles was independently performed by two investigators (W. H. and N. W.), and disagreements were resolved by consultation with a third investigator (W. X.).

2.3. Data Extraction. Two investigators (W. H. and G. Y.) independently extracted the data by using a prepared checklist, and a third investigator (W. X.) was consulted when disagreements arose. The following information was extracted from the eligible studies: the first author's name, year of publication, study design, main inclusion criteria, sample size, population demographics (including age, sex, glycosylated hemoglobin, ankle brachial index, and body mass index), characteristics of the ulcer (grade, area, and duration), information about treatments received, follow-up period, and outcomes. Incidences of the following endpoints were also extracted: completely healed ulcers, time to complete heal, reduction in the ulcer area and depth, adverse events, and quality of life. Complete healing was defined as full epithelialization. To allow an intention-to-treat analysis, the data reflecting the original allocation group were extracted. In addition, data were obtained where possible when they were published on ClinicalTrial.gov database or presented in graphical form in the articles.

2.4. Quality Assessment. The risk of bias in the included studies was assessed independently by two investigators (W. H. and C. W.), using the Cochrane Risk of Bias Assessment tool which contained the following domains: random sequence generation, allocation concealment, blinding of participants and personnel, blinding of outcome assessment, incomplete outcome data, selective reporting, and other biases. The risk of bias for each domain was assessed as either unclear, low, or high. Any discrepancies were handled by consultation with the third investigator (W. X.).

2.5. Statistical Analysis. All analyses were performed in accordance with the intention-to-treat principles. Differences in continuous outcomes (i.e., time to complete heal) are expressed as mean difference (MD) including 95\% confidence interval $(95 \% \mathrm{CI})$. Differences in dichotomous outcomes (i.e., complete healing rate) are expressed as risk ratio (RR) with $95 \% \mathrm{CI}$. Heterogeneity was estimated by the $I^{2}$ statistics. At an $I^{2} \geq 50 \%$, heterogeneity was considered as significant. A fixed effects model was used in case of low heterogeneity, and a random effects model was used if heterogeneity test revealed statistical significance. Data analyses were performed by Review Manager (RevMan) software (version: 5.3; The Cochrane Collaboration, Copenhagen, Denmark). For outcomes that were reported in $\geq 5$ studies, publication bias was assessed by Begg's test and Egger's test through STATA software (version 16.0; Stata Corp LP, College Station, TX). Sensitivity analysis was performed by deleting each individual study, using the STATA software. All statistical tests were two-sided and a $P$ value of $<0.05$ was considered significant.

\section{Results}

3.1. Literature Search. A flowchart of the literature screening process is shown in Figure 1. A total of 343 potentially relevant citations were identified, and of which, 133 citations were excluded for duplication. Then, screening of titles and abstracts resulted in the removal of 165 citations in accordance with the inclusion or exclusion criteria. After reading the full texts, 36 articles were excluded, three of them were duplicate studies [16-18], one of them was a conference abstract without available full text [19], while the other one only included 6 patients in the control group [20]. Finally, 9 RCTs were eligible for this metaanalysis [21-29].

3.2. Study Characteristics. The characteristics of the included studies are presented in Table 1. A total of 897 patients with DFU were included, 469 receiving AM therapy plus ST and 


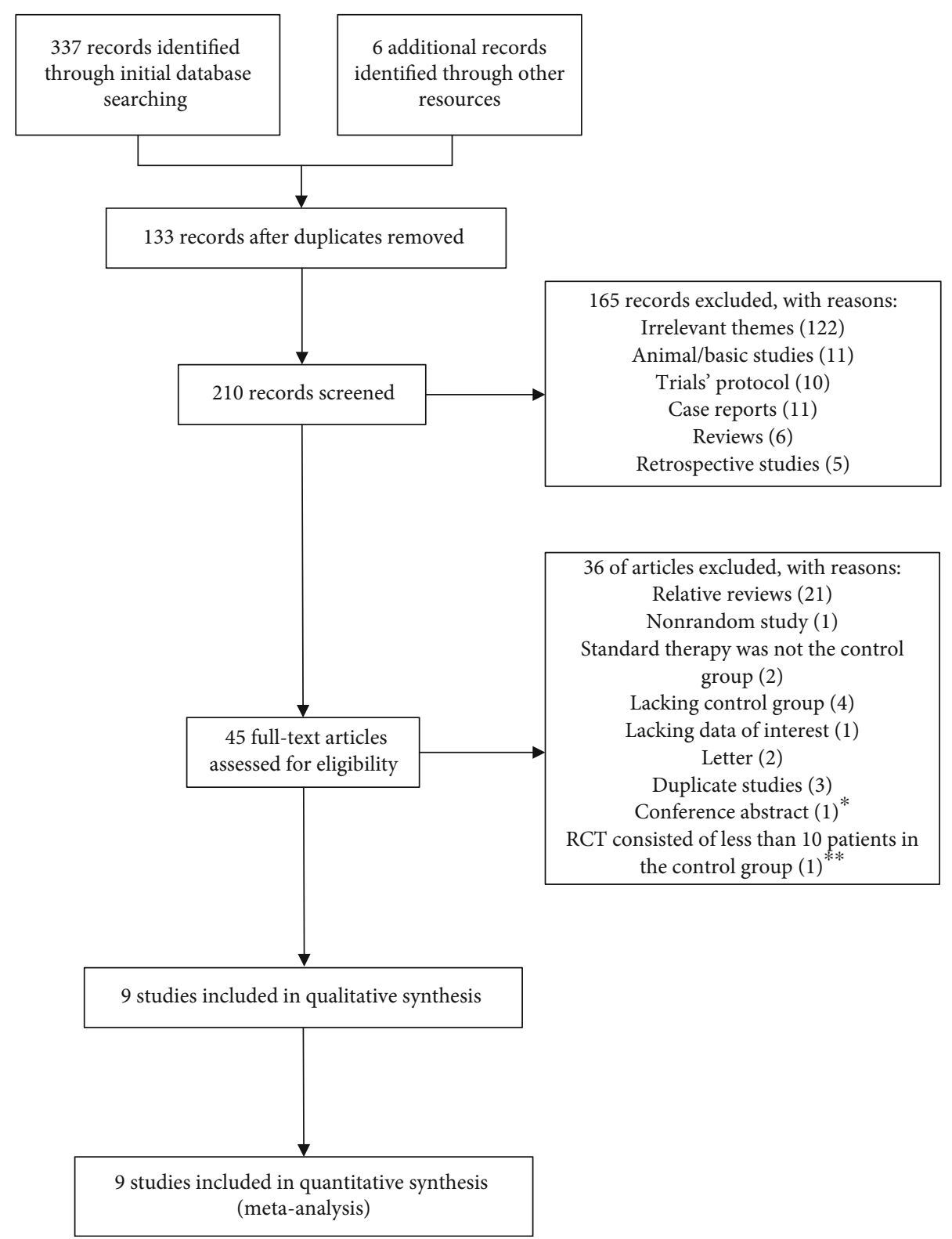

FIGURE 1: Flow chart of the study selection. * The article was a conference abstract without available full text and lacked enough information to conduct a quality assessment. **The RCT only included 6 patients in the control group.

428 receiving merely ST. The most commonly used ST included debridement, offloading, dressing, and antibiotic treatment. The main entry criteria for the different studies were similar, as shown in Table 1(a). Obese elderly patients accounted for the majority of the recruited population in most studies, with an average age of more than 55 years and an average BMI of more than $28 \mathrm{~kg} / \mathrm{m}^{2}$. Patients usually had an adequate circulation to the effected extremity. The great majority of the included foot ulcers were full thickness which corresponded to Wagner grades 1 and 2 or University of Texas grades 1-2, noninfective, chronic and refractory, while DFUs in one study [27] were Wagner grade 3. Sample sizes ranged from 14 to 154 patients and follow-up period varied between 4 weeks and 28 weeks.
3.3. Quality Assessment. The results of the bias assessment are presented in Figure 2. All studies were described as RCTs, of which 5 studies [24, 26-29] clearly described the method of random sequence generation and 2 studies $[26,28]$ reported allocation concealment. Although the blinding method of participants and personnel were not mentioned in 3 studies $[22,23,27]$, all 9 studies were assessed as having a high risk of performance bias, as AM was easily to be identified during the application by the study staff. Blinding of outcome assessment was reported in only 4 of the studies [21, 26, 28, 29], and most studies were considered to have a low risk of attrition bias and reporting bias.

3.4. Clinical Results. The incidence of complete healed ulcers was the primary outcome. Time to complete heal, ulcer area 


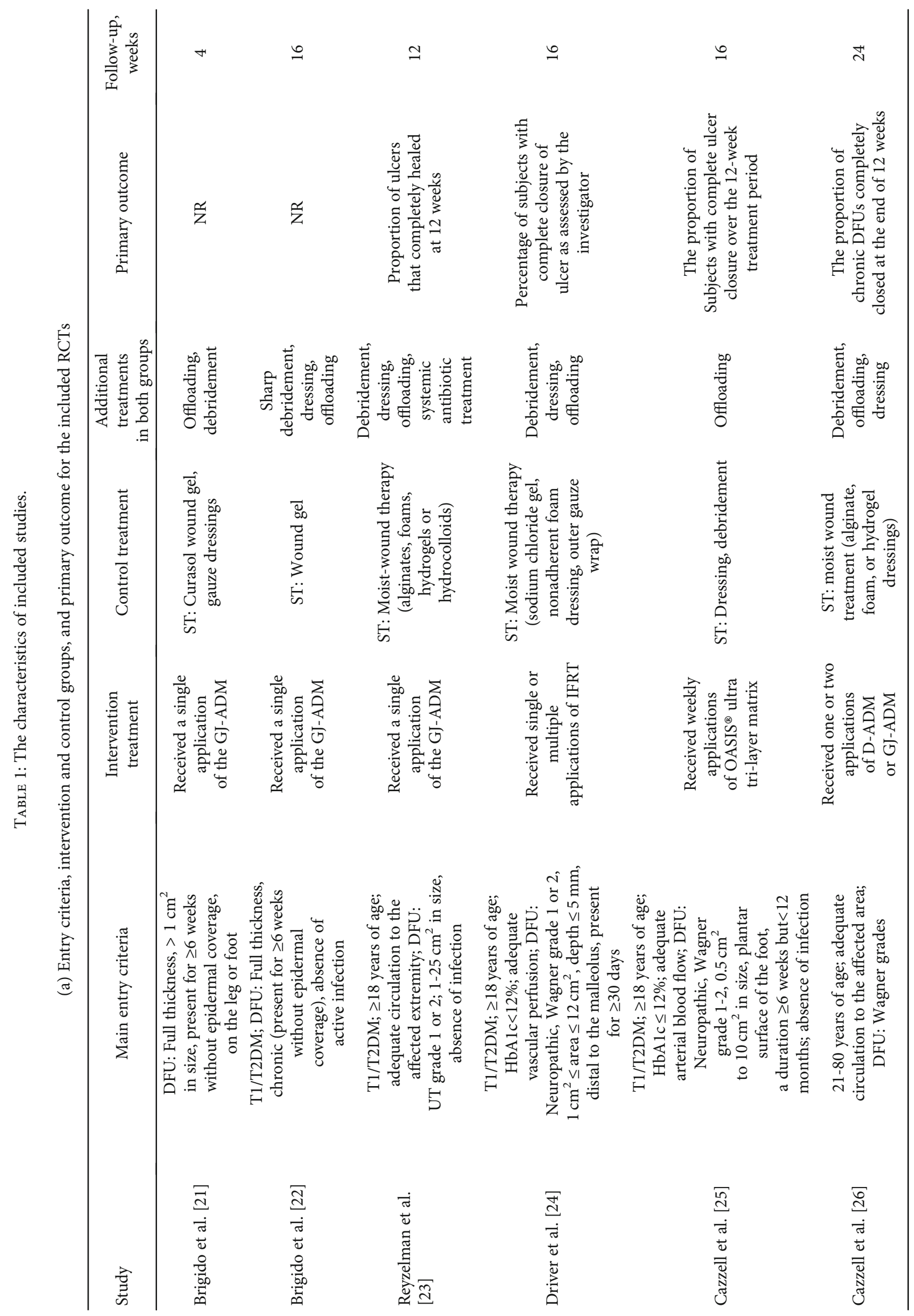




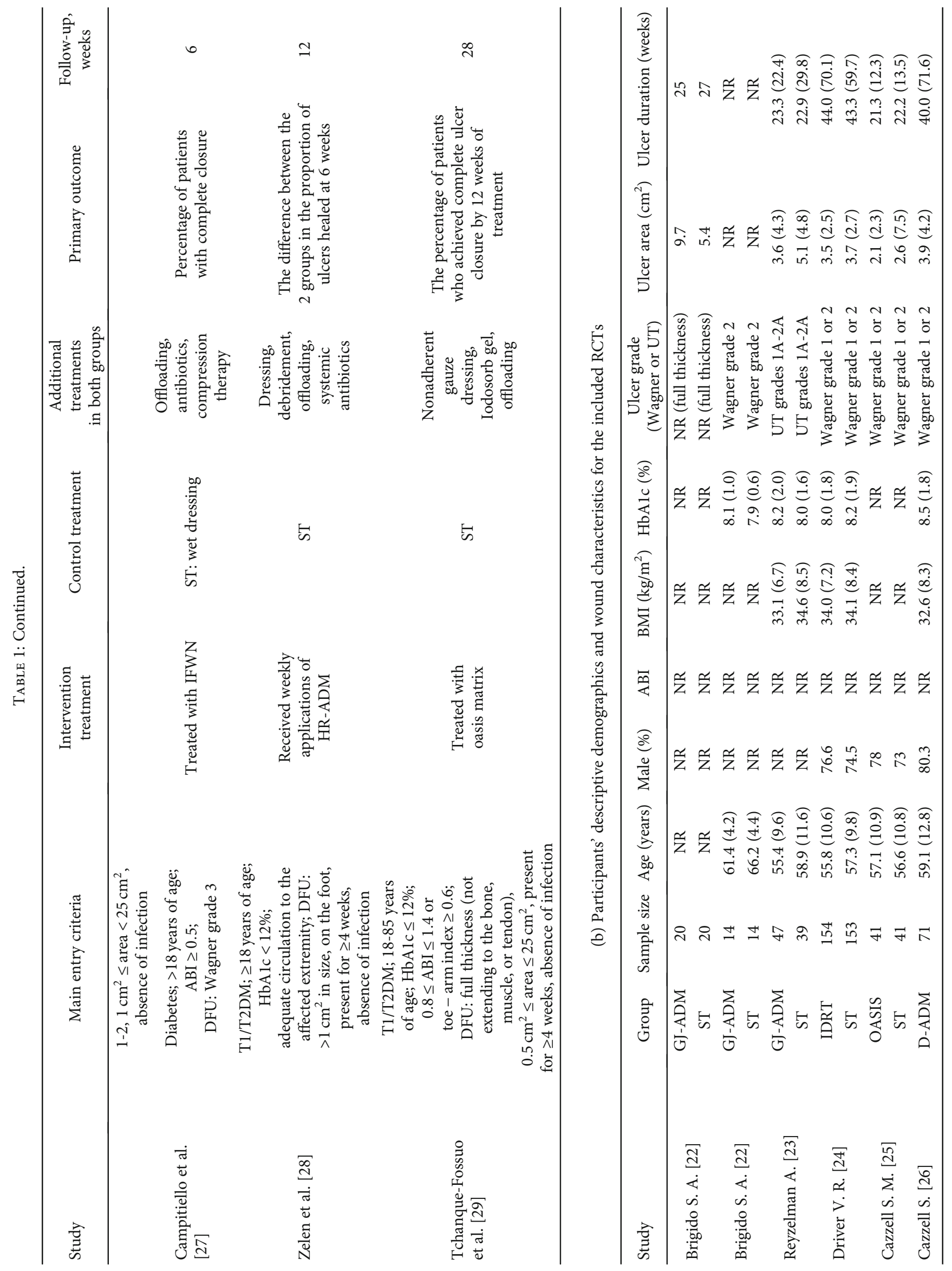




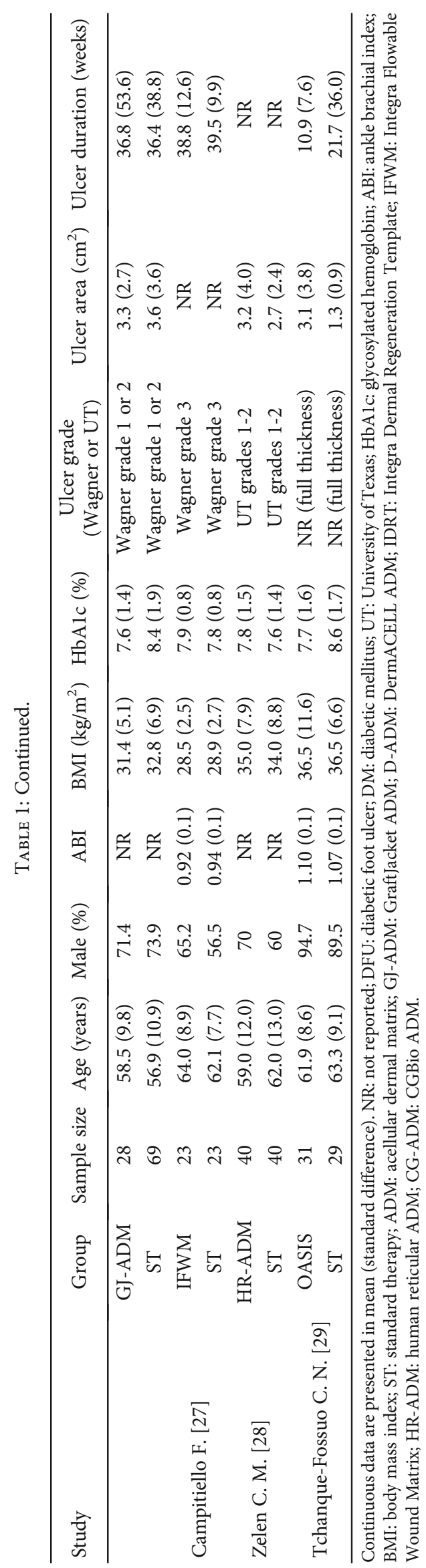




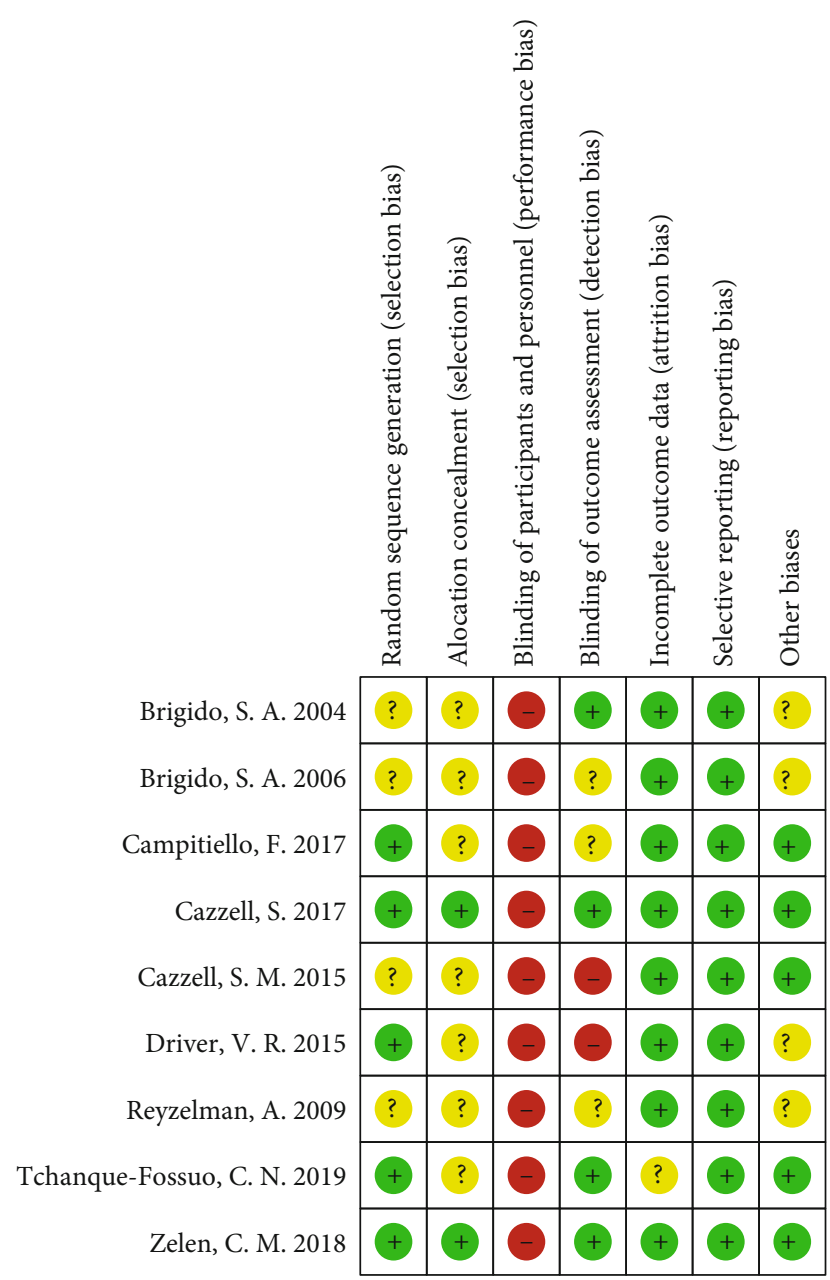

FIGURE 2: Risk of bias summary.

reduction, ulcer depth reduction, adverse events, and quality of life served as secondary outcomes.

\subsubsection{Complete Healing Rate}

(1) Complete Healing Rate at 12 Weeks. There were 7 studies $[22-26,28,29]$ involving 810 patients who reported the incidence of complete healed ulcers in 12 weeks, with 425 patients randomized to receive AM therapy and 385 patients randomized to receive ST. After 12 weeks of treatments, the complete healing rate in the AM group was higher than that in the ST group $(\mathrm{RR}=1.73,95 \% \mathrm{CI}$ : 1.31 to $2.30, P=0.0001$; Figure 3 ), using a random effects model $\left(I^{2}=54 \%\right)$.

(2) Complete Healing Rate at 16 Weeks. Four studies [22, 24-26] involving 585 patients reported the complete healing rate at 16 weeks, 308 patients and 277 patients were randomly assigned to the AM group and ST group, respectively. The pooled result showed that complete healing rate at 16 weeks in the AM group was also higher than that in the ST group $(\mathrm{RR}=1.56,95 \% \mathrm{CI}: 1.28$ to 1.91, $P<0.00001 ; I^{2}=18 \%$; Figure 4 ).
(3) Complete Healing Rate at 6 Weeks and 28 Weeks. Only 3 studies $[22,27,28]$ involving 154 patients reported the incidence of complete healed ulcers in 6 weeks, with 77 patients randomized to each group. No significant differences existed between the two groups ( $\mathrm{RR}=2.62,95 \% \mathrm{CI}: 0.88$ to 7.84 , $P=0.08 ; \mathrm{I}^{2}=84 \%$; Figure 5). In one study [29] consisting of 12-week active phase and 16-week follow-up phase, there was no significant difference $(P=0.297)$ in the complete healing rate between the AM group (48.4\%) and the ST group (48.3\%) at 28 weeks.

3.4.2. Time to Complete Heal. Five studies [22-24, 27, 28] involving 546 patients reported suitable data of complete healing time and were included in this meta-analysis. As heterogeneity test revealed statistical significance $\left(I^{2}=74 \%\right)$, a random effects model was used. The complete healing time in the AM group was shorter than that in the ST group $(\mathrm{MD}=-2.41$; $95 \%$ CI: -3.49 to $-1.32, P<0.0001$; Figure 6$)$.

3.4.3. Adverse Events. All studies reported adverse events, and only the adverse events related to AM and DFU were pooled. Most of them were diabetic foot infection, amputation, and seroma. The adverse events in the AM group were fewer than that in the ST group ( $\mathrm{RR}=0.64,95 \% \mathrm{CI}: 0.44$ to 0.93 , $P=0.02 ; I^{2}=18 \%$; Figure 7$)$.

3.4.4. Ulcer Depth Reduction/Ulcer Area Reduction/Quality of Life. One study [21] reported that there was a significant $(P<0.001)$ difference between the AM group $(89.1 \%)$ and the ST group (25\%). Two studies $[21,28]$ reported the mean reduction in ulcer area which ranged from $62 \%$ to $73.1 \%$ in the AM group and $34.2 \%$ to $52 \%$ in the ST group. Quality of life was evaluated in two studies. One study [26] involving 168 patients reported that there were no significant differences between the two groups for the total source or any of the eight areas, assessed by the SF-36 v2.0 (Optum, Inc.). However, another [24] showed significant improvements in physical functioning and bodily pain for the AM group, using the same evaluation scale.

3.5. Sensitivity Analysis. We performed sensitivity analysis by omitting one study in each turn and re-estimating the outcome. Sensitivity analysis did not identify any marked difference in the relative risk and mean difference with respect to complete healing rate at 12 weeks and 16 weeks, and time to complete heal, indicating good reliability of the outcomes. The pooled results of adverse events were as follows: $\mathrm{RR}=$ $0.64,95 \%$ CI: 0.44 to $0.93, P=0.02 ; I^{2}=18 \%$. However, as shown in Figure 8, the results changed when the study of Campitiello et al. [27] was removed: $\mathrm{RR}=0.84,95 \% \mathrm{CI}$ : 0.55 to $1.26, P=0.39 ; I^{2}=0 \%$.

3.6. Publication Bias. The publication bias was assessed by Egger's test and Begg's test. As shown in Table 2, the $P$ values of Egger's test and Begg's test were all greater than 0.05 for ulcer complete healing rate at 12 weeks, time to heal, and adverse events, indicating no significant evidence of publication bias existed. 


\begin{tabular}{|c|c|c|c|c|c|c|c|c|c|}
\hline \multirow{2}{*}{ Study or subgroup } & \multicolumn{2}{|c|}{ Acellular matrix } & \multicolumn{2}{|c|}{ Standard therapy } & \multirow{2}{*}{ Weight } & Risk ratio & \multicolumn{2}{|c|}{ Risk ratio } & \\
\hline & Events & Total & Events & Total & & M-H, random, 95\% CI & \multicolumn{2}{|c|}{ M-H, random, 95\% CI } & \\
\hline Brigido, S.A.2006 & 9 & 14 & 2 & 14 & $3.8 \%$ & $4.50[1.18,17.21]$ & & & \\
\hline Cazzell, S. 2017 & 37 & 99 & 23 & 69 & $17.3 \%$ & $1.12[0.74,1.71]$ & & & \\
\hline Cazzell, S. M. 2015 & 22 & 41 & 13 & 41 & $14.0 \%$ & $1.69[0.99,2.88]$ & & & \\
\hline Driver, V. R. 2015 & 70 & 154 & 31 & 153 & $19.4 \%$ & $2.24[1.57,3.21]$ & & & \\
\hline Reyzelman, A. 2009 & 32 & 46 & 18 & 39 & $18.3 \%$ & $1.51[1.02,2.22]$ & & & \\
\hline Tchanque-Fossuo, C. N. 2009 & 14 & 31 & 11 & 29 & $12.2 \%$ & $1.51[1.02,2.22]$ & & & \\
\hline Total $(95 \% \mathrm{CI})$ & & 425 & & 385 & $100.0 \%$ & $1.73[1.31,2.30]$ & & & \\
\hline \multirow{3}{*}{\multicolumn{5}{|c|}{$\begin{array}{l}\text { Total events } \\
\text { Heterogeneity: } \operatorname{tau}^{2}=0.07 ; \mathrm{chi}^{2}=13.03, \mathrm{df}=6(P=0.04) ; I^{2}=54 \% \\
\text { Test for overall effect: } Z=3.85(P=0.0001)\end{array}$}} & & & & & \\
\hline & & & & & & 0.05 & 0.2 & 5 & 20 \\
\hline & & & & & & & Favours ST & Favours AM & \\
\hline
\end{tabular}

Figure 3: Forest plot of complete healing rate at 12 weeks.

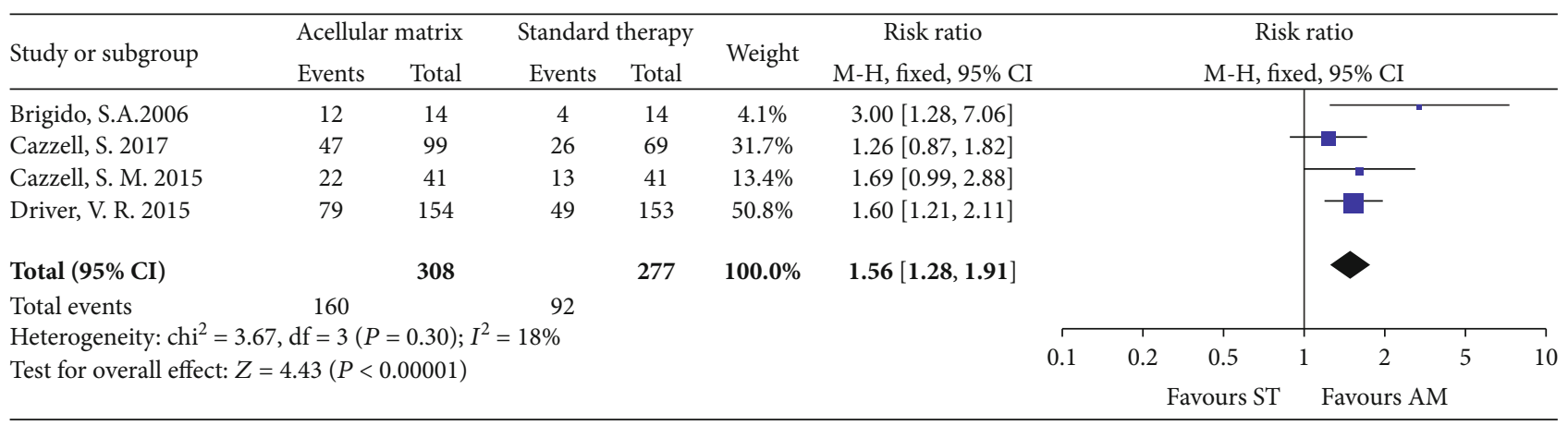

FIGURE 4: Forest plot of complete healing rate at 16 weeks.

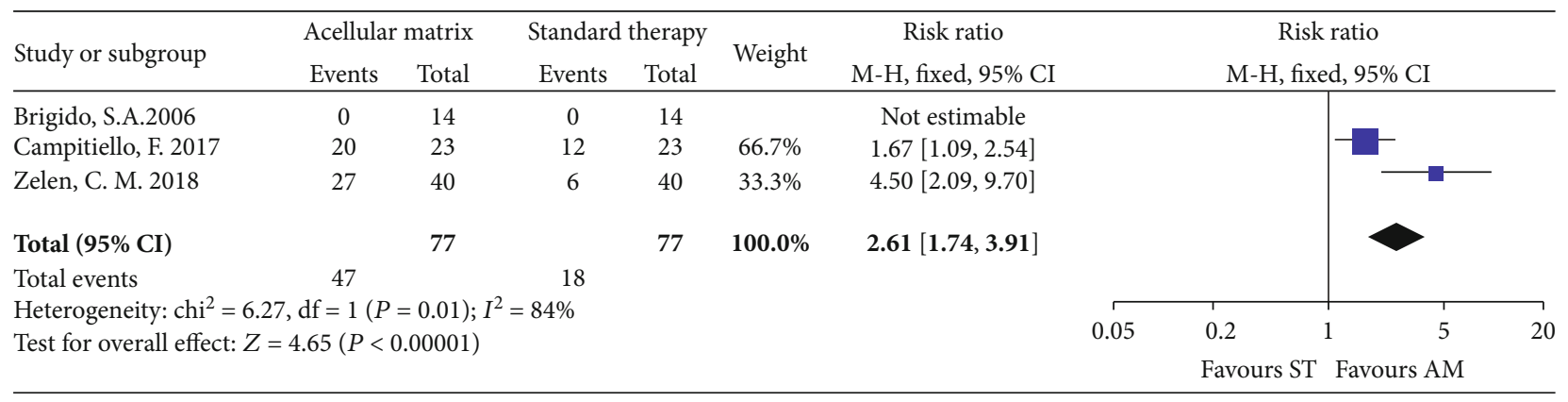

FIGURE 5: Forest plot of complete healing rate at 6 weeks.

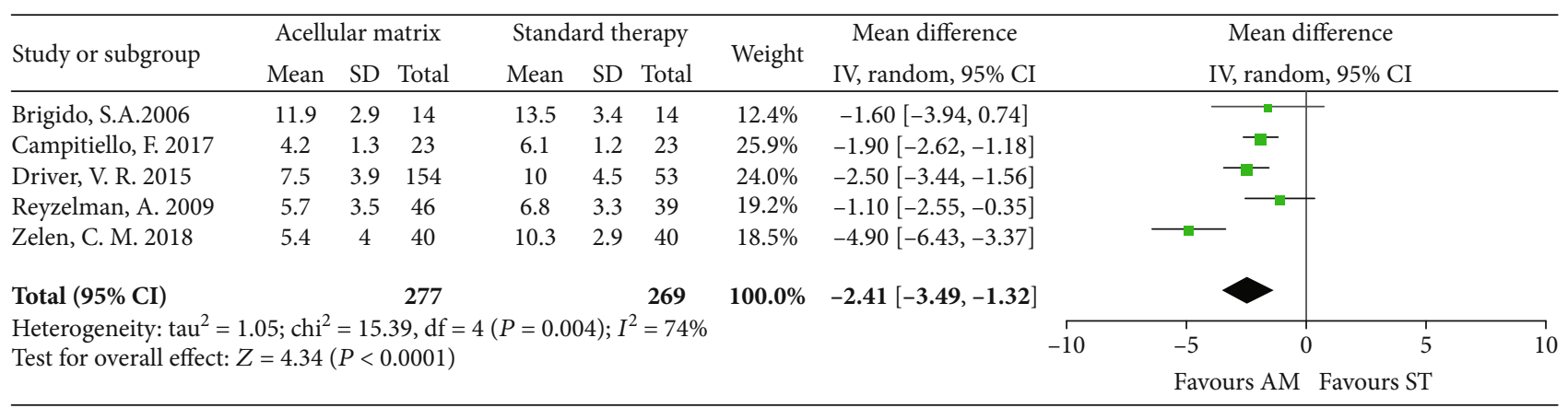

FIgURE 6: Forest plot of time to complete heal. 


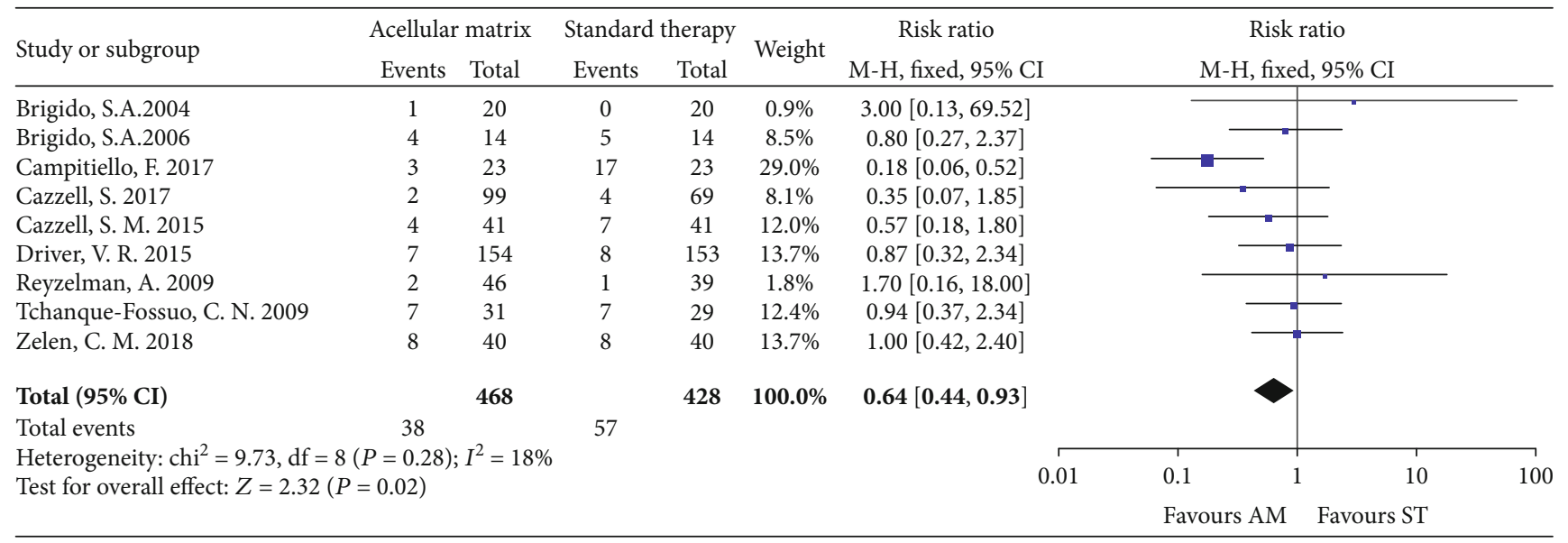

Figure 7: Forest plot of adverse events.

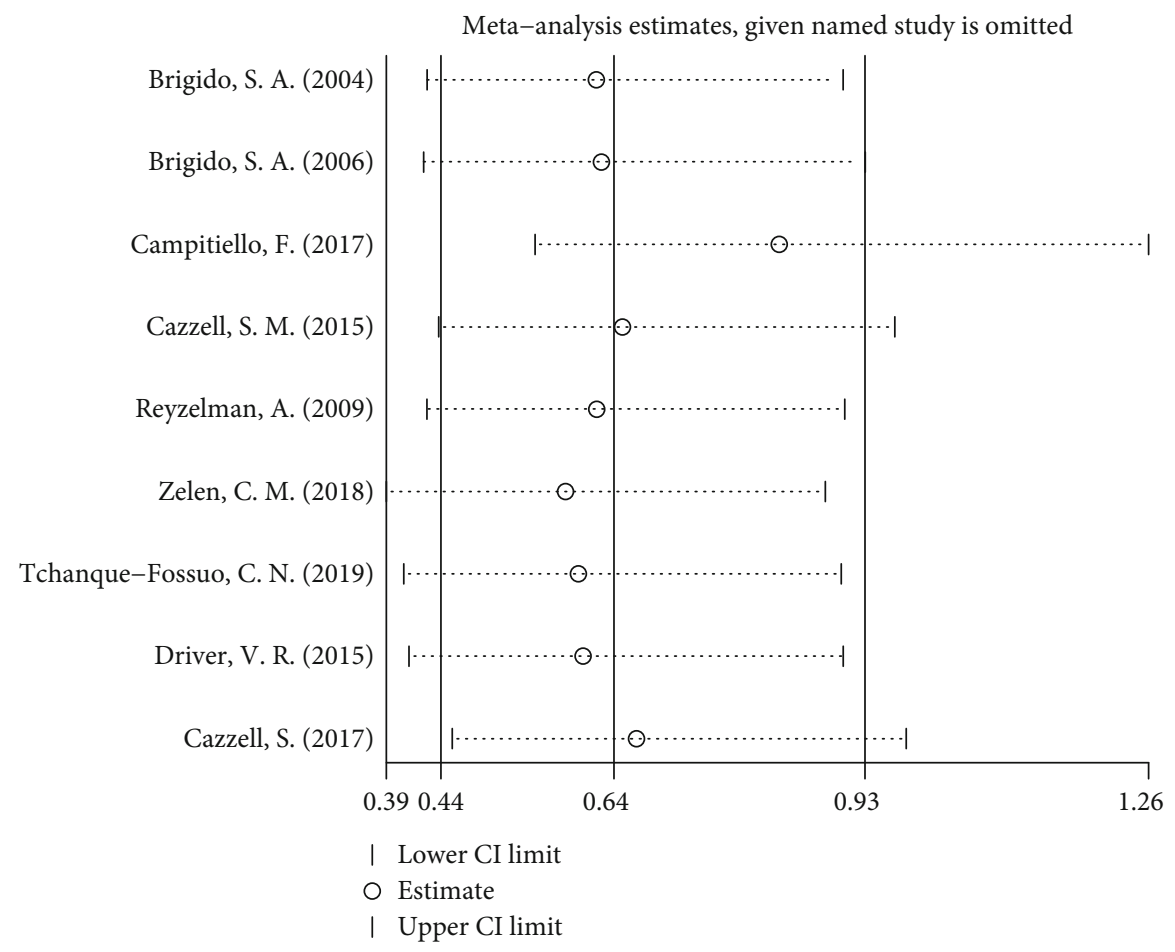

FIGURE 8: Sensitivity analysis of adverse events.

TABLE 2: Egger's test and Begg's test for the outcomes.

\begin{tabular}{lcc}
\hline Outcome & \multicolumn{2}{c}{$\mathrm{P}$} \\
& Egger's test & Begg's test \\
\hline Complete healing rate at 12 weeks & 0.617 & 1.000 \\
Time to complete heal & 0.438 & 0.806 \\
Adverse events & 0.766 & 0.754 \\
\hline
\end{tabular}

\section{Discussion}

In the present study, we performed a meta-analysis of 9 RCTs involving 897 patients and evaluated the efficacy and safety of AM therapy for DFUs. It was found that AM therapy was sig- nificantly associated with a higher complete healing rate at 12 weeks and 16 weeks, a shorter complete healing time, and fewer adverse events.

To our knowledge, this is the first meta-analysis to evaluate the effectiveness and safety profile of AM for patients with DFUs. Reyzelman et al. [10] performed a quantitative analysis of 3 RCTs to estimate the effectiveness of one specific human acellular dermal matrix (ADM; Graftjacket regenerative tissue matrix) in healing DFUs. Xue et al. [30] conducted a meta-analysis of 5 RCTs and assessed the efficacy and safety of allogenic ADM for DFUs. A recent meta-analysis of 6 RCTs [31] suggested that compared with the merely ST, patients in ADM group had a higher complete healing rate 
and faster time to heal. Moreover, no significant difference existed in adverse events between both groups. Different from our study, the studies mentioned above only involved one or more different varieties of ADM, which are a part of AMs. As we know, the AMs are derived not only from human and animal skin, called ADM, but also from other tissues, such as porcine small intestinal submucosa, urinary bladder matrix, and pericardium $[13,14]$. The second difference is that two RCTs $[16,17]$ included in Guo's study were ongoing and only partial results were analyzed, whereas we incorporated the complete results of these 2 RCTs $[26,28]$. The larger sample size probably enhanced the power of analysis.

In our meta-analysis, the results revealed that AM therapy could promote the healing of DFUs. The likelihood of ulcer complete healing in AM group is 1.73 and 1.56 times more than the ST group at 12 weeks and 16 weeks, respectively. On the basic of ST, AM therapy could further shorten the complete healing time for patients with DFUs $(\mathrm{MD}=-2.41 ; 95 \% \mathrm{CI}:-3.49$ to -1.32$)$. These finding are robust as sensitivity analysis had confirmed that omitting any study would not change the direction of the outcomes. Considering that the estimation of the complete healing time is largely based on the trend of complete healing rate and thus influenced by the different durations of follow-up, we also analyzed this outcome separately according to the follow-up duration. The pooled results still supported the above conclusion. For the outcome of complete healing rate at 6 weeks, it was shown that between the two groups, no significant difference existed. Here, only 2 studies $[27,28]$ were analyzed while another [22] reported that no ulcers completely healed in 6 weeks. The heterogeneity was high, and it might result from the different severities of ulcers and different AM products. Therefore, we should look at this result with caution. It may indicate that it is difficult to show a great superiority of AM therapy over ST in a relatively short period of time. After all, DFUs usually take a long time to be cured. Several studies have shown that the mean or median healing time ranged from 2 to 4 months, even for those treated with AMs [32-35]. Further studies are needed to explore the short-term effects of AMs.

In the wound care community, concerns have been expressed that AM therapy might lead to more adverse events, such as infection and graft rejection $[13,36]$. Our meta-analysis shows that people in the AM group have fewer adverse events compared to those in ST group ( $R R=0.64$, $95 \%$ CI: 0.44 to $0.93, P=0.02$ ). To be worthy of attention, only 1 of 9 studies reported a significant difference of adverse events between the two groups. When the study [27] was removed, the heterogeneity decreased from $18 \%$ to $0 \%$, and the pooled result changed: $\mathrm{RR}=0.84,95 \%$ CI: 0.55 to $1.26, P=0.39$. This may be explained by the different severities of ulcers. In the study of Campitiello et al., the ulcers with grade 3 Wagner classification were more serious than those in other studies. However, the study was not dropped from the meta-analysis for the following reasons: (1) it satisfied all the inclusion criteria and was not related to the exclusion criteria; (2) reserving this study could bring our meta-analysis closer to reality, con- sidering that there are a considerable part of DFUs classified as Wagner grade 3 in clinical practice. In another view, our meta-analysis at least proves that AM therapy would not increase the incidence of adverse events, indicating a safety profile.

Endpoints such as ulcer area reduction and ulcer depth reduction are the clinically relevant outcomes reflecting the effectiveness of AM therapy. Although some studies [21, 26, 28] reported that participants in the AM group had more reduction in the ulcer area or depth than those in the ST group, we could not conduct a meta-analysis due to the lack of suitable data. Other endpoints such as quality of life and cost-effectiveness also need to be considered. In this metaanalysis, only 2 RCTs $[24,26]$ evaluated the quality of life and their conclusions were not consistent. Additionally, while AM therapy leads to higher economic costs, the potential savings associated with accelerated closure of these chronic, refractory DFUs should be considered. It was disappointing that no RCTs compare the cost-effectiveness of AM therapy with ST. Researchers need to pay attention to these outcomes in their further studies.

In our meta-analysis, the quality of included clinical trials is middle to excellent and the strength of evidence is moderate. However, a few limitations should be acknowledged. First, although a comprehensive literature search was conducted and Begg's test or Egger's test revealed no publication bias existed, some negative outcomes might not be published because these studies were always related to commercial corporations. And only 2 studies $[25,29]$ assessing the same AM product derived from other tissues rather than from the skin were included. Second, the differences in AM products and standard therapies in each study may have affected our outcomes. Third, although there were 9 RCTs included, the sample size of each study was relatively small. For some outcomes, the limited number of studies were included and analyzed. Fourth, the criterion of adverse events was not very clear in some of the trials. Fifth, the included patients usually had adequate perfusion and a great majority of foot ulcers was full thickness and noninfective; thus, our findings may not be applicable to all patients with DFUs. Finally, due to the short-term follow-up duration of all studies, we could not explore the long-term effects of AM therapy.

\section{Conclusion}

In conclusion, the present meta-analysis suggests that AM therapy as an adjuvant treatment could further promote the healing of full-thickness, noninfected, and nonischemic diabetic foot ulcers. AM therapy also has a safety profile. However, because of various limitations, more large welldesigned randomized clinical trials with long follow-up duration are needed to further explore the efficacy and safety of AM therapy for DFUs.

\section{Data Availability}

The data supporting this meta-analysis are from previously reported studies, which have been cited. The processed data are available from the corresponding author upon request. 


\section{Conflicts of Interest}

The authors declare that there is no conflict of interest regarding the publication of this paper.

\section{Acknowledgments}

This work was supported by the National Natural Science Foundation of China (nos. 30971665, 81172894, and 81370925); the Natural Science Foundation of Guangdong Province (nos. S2012010009336 and 10151503102000017); and the Science and Technology Planning Project of Guangdong Province (no. 2013B021800254).

\section{Supplementary Materials}

The PRISMA 2009 checklist is provided. (Supplementary Materials)

\section{References}

[1] B. Zhou, Y. Lu, K. Hajifathalian et al., "Worldwide trends in diabetes since 1980: a pooled analysis of 751 population- based studies with $4 * 4$ million participants," The Lancet, vol. 387, no. 10027, pp. 1513-1530, 2016.

[2] N. H. Cho, J. E. Shaw, S. Karuranga et al., "IDF diabetes atlas: global estimates of diabetes prevalence for 2017 and projections for 2045," Diabetes Research and Clinical Practice, vol. 138, pp. 271-281, 2018.

[3] D. C. Jupiter, J. C. Thorud, C. J. Buckley, and N. Shibuya, "The impact of foot ulceration and amputation on mortality in diabetic patients. I: from ulceration to death, a systematic review," International Wound Journal, vol. 13, no. 5, pp. 892-903, 2016.

[4] P. A. Lazzarini, R. E. Pacella, D. G. Armstrong, and J. J. van Netten, "Diabetes-related lower-extremity complications are a leading cause of the global burden of disability," Diabetic Medicine, vol. 35, no. 9, pp. 1297-1299, 2018.

[5] H. Tchero, P. Kangambega, L. Lin et al., "Cost of diabetic foot in France, Spain, Italy, Germany and United Kingdom: a systematic review," Annales d'Endocrinologie, vol. 79, no. 2, pp. $67-74,2018$.

[6] P. Zhang, J. Lu, Y. Jing, S. Tang, D. Zhu, and Y. Bi, "Global epidemiology of diabetic foot ulceration: a systematic review and meta-analysis ${ }^{\dagger}, "$ Annals of Medicine, vol. 49, no. 2, pp. 106116, 2016.

[7] D. G. Armstrong, A. J. M. Boulton, and S. A. Bus, "Diabetic foot ulcers and their recurrence," The New England Journal of Medicine, vol. 376, no. 24, pp. 2367-2375, 2017.

[8] E. Everett and N. Mathioudakis, "Update on management of diabetic foot ulcers," Annals of the New York Academy of Sciences, vol. 1411, no. 1, pp. 153-165, 2018.

[9] D. J. Margolis, J. Kantor, and J. A. Berlin, "Healing of diabetic neuropathic foot ulcers receiving standard treatment. A metaanalysis," Diabetes Care, vol. 22, no. 5, pp. 692-695, 1999.

[10] A. M. Reyzelman and I. Bazarov, "Human acellular dermal wound matrix for treatment of DFU: literature review and analysis," Journal of Wound Care, vol. 24, no. 3, pp. 128-134, 2015.

[11] N. S. Greaves, S. A. Iqbal, M. Baguneid, and A. Bayat, "The role of skin substitutes in the management of chronic cutaneous wounds," Wound Repair and Regeneration, vol. 21, no. 2, pp. 194-210, 2013.

[12] R. S. Kirsner, G. Bohn, V. R. Driver et al., "Human acellular dermal wound matrix: evidence and experience," International Wound Journal, vol. 12, no. 6, pp. 646-654, 2015.

[13] M. Climov, L. R. Bayer, A. V. Moscoso, H. Matsumine, and D. P. Orgill, "The role of dermal matrices in treating inflammatory and diabetic wounds," Plastic and Reconstructive Surgery, vol. 138, pp. 148s-157s, 2016.

[14] H. Cho, M. R. Blatchley, E. J. Duh, and S. Gerecht, "Acellular and cellular approaches to improve diabetic wound healing," Advanced Drug Delivery Reviews, vol. 146, pp. 267-288, 2019.

[15] D. Moher, A. Liberati, J. Tetzlaff, D. G. Altman, and The PRISMA Group, "Preferred reporting items for systematic reviews and meta-analyses: the PRISMA statement," PLoS Medicine, vol. 6, no. 7, article e1000097, 2009.

[16] J. Walters, S. Cazzell, H. Pham, D. Vayser, and A. Reyzelman, "Healing rates in a multicenter assessment of a sterile, room temperature, acellular dermal matrix versus conventional care wound management and an active comparator in the treatment of full-thickness diabetic foot ulcers," Eplasty, vol. 16, article e10, 2016.

[17] C. M. Zelen, D. P. Orgill, T. Serena et al., "A prospective, randomised, controlled, multicentre clinical trial examining healing rates, safety and cost to closure of an acellular reticular allogenic human dermis versus standard of care in the treatment of chronic diabetic foot ulcers," International Wound Journal, vol. 14, no. 2, pp. 307-315, 2017.

[18] C. Tchanque-Fossuo, S. Dahle, H. Lev-Tov, C. Li, and R. Isseroff, "910 Cellular versus acellular matrix devices in treatment of diabetic foot ulcers: preliminary results of a comparative efficacy randomized controlled trial," Journal of Investigative Dermatology, vol. 137, no. 5, article S157, 2017.

[19] D. Shin, "The use of acellular dermal matrix paste for treatment of diabetic foot ulcer," Wound Repair and Regeneration, vol. 27, no. 3, article A17, 2019.

[20] O. M. Alvarez, T. Smith, T. W. Gilbert et al., "Diabetic foot ulcers treated with porcine urinary bladder extracellular matrix and Total contact cast: interim analysis of a randomized, controlled trial," Wounds, vol. 29, no. 5, pp. 140-146, 2017.

[21] S. A. Brigido, S. F. Boc, and R. C. Lopez, "Effective management of major lower extremity wounds using an acellular regenerative tissue matrix: a pilot study," Orthopedics, vol. 27, Supplement 1, pp. s145-s149, 2004.

[22] S. A. Brigido, "The use of an acellular dermal regenerative tissue matrix in the treatment of lower extremity wounds: a prospective 16-week pilot study," International Wound Journal, vol. 3, no. 3, pp. 181-187, 2006.

[23] A. Reyzelman, R. T. Crews, J. C. Moore et al., "Clinical effectiveness of an acellular dermal regenerative tissue matrix compared to standard wound management in healing diabetic foot ulcers: a prospective, randomised, multicentre study," International Wound Journal, vol. 6, no. 3, pp. 196-208, 2009.

[24] V. R. Driver, L. A. Lavery, A. M. Reyzelman et al., "A clinical trial of integra template for diabetic foot ulcer treatment," Wound Repair and Regeneration, vol. 23, no. 6, pp. 891-900, 2015.

[25] S. M. Cazzell, D. L. Lange, J. E. Dickerson Jr., and H. B. Slade, "The management of diabetic foot ulcers with porcine small intestine submucosa tri-layer matrix: a randomized controlled 
trial," Advances in Wound Care, vol. 4, no. 12, pp. 711-718, 2015.

[26] S. Cazzell, D. Vayser, H. Pham et al., "A randomized clinical trial of a human acellular dermal matrix demonstrated superior healing rates for chronic diabetic foot ulcers over conventional care and an active acellular dermal matrix comparator," Wound Repair and Regeneration, vol. 25, no. 3, pp. 483-497, 2017.

[27] F. Campitiello, M. Mancone, A. Della Corte, R. Guerniero, and S. Canonico, "To evaluate the efficacy of an acellular flowable matrix in comparison with a wet dressing for the treatment of patients with diabetic foot ulcers: a randomized clinical trial," Updates in Surgery, vol. 69, no. 4, pp. 523-529, 2017.

[28] C. M. Zelen, D. P. Orgill, T. E. Serena et al., "An aseptically processed, acellular, reticular, allogenic human dermis improves healing in diabetic foot ulcers: a prospective, randomised, controlled, multicentre follow-up trial," International Wound Journal, vol. 15, no. 5, pp. 731-739, 2018.

[29] C. N. Tchanque-Fossuo, S. E. Dahle, H. Lev-Tov et al., "Cellular versus acellular matrix devices in the treatment of diabetic foot ulcers: interim results of a comparative efficacy randomized controlled trial," Journal of Tissue Engineering and Regenerative Medicine, vol. 13, no. 8, pp. 1430-1437, 2019.

[30] C. L. Xue, Z. C. Hu, Z. X. Yang, and X. J. Li, "Meta-analysis on the clinical effects of allogenic acellular dermal matrix treatment for diabetic foot ulcer," Zhonghua Shao Shang Za Zhi, vol. 32, no. 12, pp. 725-729, 2016.

[31] X. Guo, D. Mu, and F. Gao, "Efficacy and safety of acellular dermal matrix in diabetic foot ulcer treatment: a systematic review and meta-analysis," International Journal of Surgery, vol. 40, pp. 1-7, 2017.

[32] K. K. Kee, H. K. R. Nair, and N. P. Yuen, "Risk factor analysis on the healing time and infection rate of diabetic foot ulcers in a referral wound care clinic," Journal of Wound Care, vol. 28, Supplement 1, pp. S4-S13, 2019.

[33] P. Ince, D. Kendrick, F. Game, and W. Jeffcoate, "The association between baseline characteristics and the outcome of foot lesions in a UK population with diabetes," Diabetic Medicine, vol. 24, no. 9, pp. 977-981, 2007.

[34] C. L. Winters, S. A. Brigido, B. A. Liden, M. Simmons, J. F. Hartman, and M. L. Wright, "A multicenter study involving the use of a human acellular dermal regenerative tissue matrix for the treatment of diabetic lower extremity wounds," Advances in Skin \& Wound Care, vol. 21, no. 8, pp. 375-381, 2008.

[35] E. Lullove, "Acellular fetal bovine dermal matrix in the treatment of nonhealing wounds in patients with complex comorbidities," Journal of the American Podiatric Medical Association, vol. 102, no. 3, pp. 233-239, 2012.

[36] N. M. Protzman and S. A. Brigido, "Recent advances in acellular regenerative tissue scaffolds," Clinics in Podiatric Medicine and Surgery, vol. 32, no. 1, pp. 147-159, 2015. 